\title{
Objetivos básicos del aprendizaje del Trabajo en Equipo en la Universidad Basic learning Teamwork objectives at the University
}

\author{
Dolores Lerís ${ }^{1}$, Fernando Vea ${ }^{1}$, M. Ángeles Velamazán ${ }^{1}$, Patricia Florentín ${ }^{2}$ \\ dleris@unizar.es, fernavea@unizar.es, mavelama@unizar.es, pfd@unizar.es \\ ${ }^{1}$ Matemática Aplicada \\ Universidad de Zaragoza \\ Zaragoza, España \\ ${ }^{2}$ Matemáticas \\ Universidad de Zaragoza \\ Zaragoza, España
}

\begin{abstract}
Resumen- En esta comunicación se presenta el método de trabajo seguido para mejorar el aprendizaje del trabajo en equipo en los primeros cursos de un grado universitario. Hemos dirigido el foco hacia las habilidades y actitudes básicas a desarrollar por los estudiantes en esa competencia interpersonal al inicio de sus estudios universitarios. Los autores proponemos que la capacitación para trabajar en equipo debería abordar inicialmente dos problemas: la responsabilidad de los miembros del equipo y la ejecución de un plan de trabajo. Analizamos la percepción de los estudiantes en relación a los factores y comportamientos que los modelos de la competencia estiman más adecuados para abordarlos. Además, teniendo en cuenta los resultados de ese análisis, se ofrecen algunas sugerencias prácticas para los diseños instruccionales del trabajo en equipo.
\end{abstract}

\section{Palabras clave: trabajo en equipo, responsabilidad individual, plan de trabajo}

Abstract- This paper presents the procedure we have used to improve the teamwork learning activities in the first year of the bachelor's degree. The focus was placed on the basic teamwork skills and attitudes that should be developed by students at the beginning of their university studies. We propose that the initial teamwork training is oriented towards solving two problems: the responsibility of teammates and the effective implementation of a work plan. We analyse the point of view of the student about factors and behaviours that teamwork competence definition suggests as more appropriate to address them. Moreover, we make a certain number of recommendations for the teamwork instructional design based on the findings of this study.

Keywords: teamwork, individual responsability, task plan

\section{INTRODUCCIÓN}

Para definir una competencia es necesario describir "los conocimientos, habilidades y actitudes, que de forma combinada entran en acción para responder a las demandas que una persona ha de afrontar" (Florentín, 2016). El trabajo en equipo, en particular, es una competencia interpersonal compleja, con múltiples dimensiones, y es, por ello, que son muchas las propuestas de definición que aparecen en la literatura científica.

En este trabajo hemos utilizado como referentes el modelo propuesto por Lerís, Fidalgo y Sein-Echaluce (2014) para la formación de la competencia de trabajo en equipo en el contexto educativo y, por otro, el marco teórico adoptado por Florentín (2016) para analizar la formación ofrecida en dicha competencia en un grado universitario. En ambos casos se aprecia la influencia del modelo Inputs-Mediators-Outputs (en adelante, IMO) del contexto laboral, que formula las fases o etapas que atraviesa un equipo y que describe los factores y comportamientos que mejoran su eficacia (Mathieu, Maynard, Rapp \& Gilson, 2008; Rousseau, Aubé \& Savoie, 2006; Torrelles et al., 2011). Se trata, por tanto, de aproximaciones a la definición de la competencia de trabajo que inciden en la acción del equipo, que es, en definitiva, donde se ponen en evidencia las habilidades y actitudes propias de esta competencia interpersonal.

Esquemáticamente, el marco teórico de este estudio establece tres tipos de factores a tener en cuenta: los de entrada, los mediadores y los de salida. Entre los primeros, se encuentran condicionantes iniciales de distintos niveles: la titulación, la asignatura y el equipo; entre los mediadores, se consideran los procesos de transición y los de relación durante el desarrollo de las tareas del equipo; y, finalmente, los resultados son los que conforman la última etapa, las salidas.

Una vez realizado el primer paso, el de adoptar un modelo que guíe el diseño formativo de nuestros jóvenes universitarios en la competencia de trabajo en equipo, es esencial convenir que el desarrollo de las habilidades y actitudes propias de esta competencia interpersonal se ha de hacer de forma continua y progresiva. Y, por tanto, es ineludible planificar esa progresividad.

El método de actuación seguido por los autores para mejorar el diseño del aprendizaje de la competencia de trabajo en equipo al inicio de los estudios universitarios, se puede esquematizar en tres fases. En primer lugar, se ha realizado una reflexión y propuesta de los aspectos básicos ligados a la competencia de trabajo en equipo; que se ha acompañado, después, de una consulta de la percepción del alumnado al respecto y, finalmente, se incorporan los resultados, a modo de recomendaciones, en el diseño instruccional.

A continuación, en la sección del contexto, explicamos cuáles son, a nuestro juicio, las habilidades básicas que han de desarrollar nuestros estudiantes al inicio de su formación universitaria en relación a la competencia de trabajo en 
equipo. En la sección de descripción, detallamos el proceso de recogida de información sobre la percepción de los estudiantes en relación a esas habilidades básicas. El análisis de los datos obtenidos es expuesto en la sección de resultados. Y, finalmente, en las conclusiones explicamos brevemente las recomendaciones respecto al consecuente diseño instruccional.

\section{CONTEXTO}

Al comienzo de los estudios universitarios, dos son los problemas que consideramos que es prioritario abordar en relación al trabajo en equipo: el de la responsabilidad de los miembros del equipo y el de la ejecución de un plan de trabajo. El modelo IMO ofrece algunas respuestas a esos problemas, en diferentes fases y a través de los factores que se comentan a continuación.

Respecto a la responsabilidad de los miembros del equipo, dos factores de entrada (Inputs) destacan como posibles soluciones. A nivel de asignatura, la evaluación de los trabajos en equipo determina el esfuerzo del grupo y del individuo. Y, a nivel de equipo, el método y los criterios para su constitución afectan a la disposición para colaborar y a su cohesión. Además, hemos considerado un factor mediador (Mediators), que forma parte de los procesos operativos de transición: el establecimiento de normas de funcionamiento del equipo y, en particular, la regulación del no cumplimiento de los compromisos grupales.

En cuanto a la ejecución de un plan de trabajo, es un asunto arduo, que, según nuestra experiencia, es el causante de grandes dificultades en la ejecución exitosa de la tarea. Los factores mediadores (Mediators) que hemos considerado en este estudio son algunos de los que se califican como procesos operativos de relación. En concreto, nos referimos a los siguientes:

- $\quad$ La participación equitativa.

- La toma de decisiones.

- $\quad$ El seguimiento interno de las tareas (coordinador).

- El seguimiento externo de las tareas (profesorado).

Una vez establecidos los problemas formativos básicos de la competencia de trabajo en equipo, y los factores y comportamientos ligados a ellos, el siguiente objetivo del estudio es analizar la percepción de los estudiantes, según su experiencia universitaria. Para completar el estudio, también optamos por indagar sobre los factores de salida (Outputs) o resultados de aprendizaje con los trabajos en equipo.

El público objetivo de este estudio son alumnos de segunda matrícula, o más, en la asignatura de Matemáticas I de los Grados de Ingeniería de la Escuela de Ingeniería y Arquitectura de la Universidad de Zaragoza, y que, además, han optado por cursarla en el grupo de docencia que el Centro ofrece en un cuatrimestre diferente (el segundo en este caso) al establecido en el Plan de Estudios.

\section{DESCRIPCIÓN}

El estudio realizado es exploratorio, basado en las respuestas de los estudiantes a un cuestionario, elaborado a partir del cuestionario ACOES (García, González y Mérida, 2012), para indagar sobre los aspectos básicos de la competencia de trabajo en equipo mencionados en la sección anterior.
Dicho cuestionario contiene un primer bloque de preguntas para conocer algunas características del participante: año de nacimiento (P1), género (P2), años en la Universidad (P3), número de trabajos en equipo realizados (P4), y nivel de importancia (P5) y dominio de la competencia (P6) de trabajo en equipo. Y otro bloque de preguntas (de la 7 a la 12) ya relacionado con el objeto directo de este estudio: habilidades básicas en el trabajo en equipo; los enunciados aparecen en la sección de resultados.

El cuestionario fue implementado en la plataforma Moodle y fue respondido por 71 de los 78 estudiantes de la asignatura (91.03\%) durante el mes de marzo de 2017.

Las características de los participantes son las siguientes:

- Género: $72 \%$ hombres y 28\% mujeres.

- $\quad$ Años en la Universidad: para el $77.5 \%$ es su segundo año en la Universidad, para el $19.7 \%$ es el tercero y para el $2.8 \%$ restante es su cuarto año.

- $\quad$ Número de trabajos durante los estudios universitarios: el 75\% de los participantes declara haber realizado entre 3 y 12 trabajos y el 25\% restante hasta 25 como máximo.

- $\quad$ El 99\% de los participantes opinan que las habilidades para trabajar en equipo son bastante o muy importantes en la profesión para la que se están formando. Si bien, por cada uno que afirma que es muy importante, hay 1.7 que la califican únicamente como bastante importante (véase la figura 1).

- $\quad$ Sin embargo, el 87\% declaran que su nivel de dominio de las habilidades para trabajar en equipo es bastante alto y tan sólo el 6\% expresan que su nivel es muy alto (véase la figura 1).

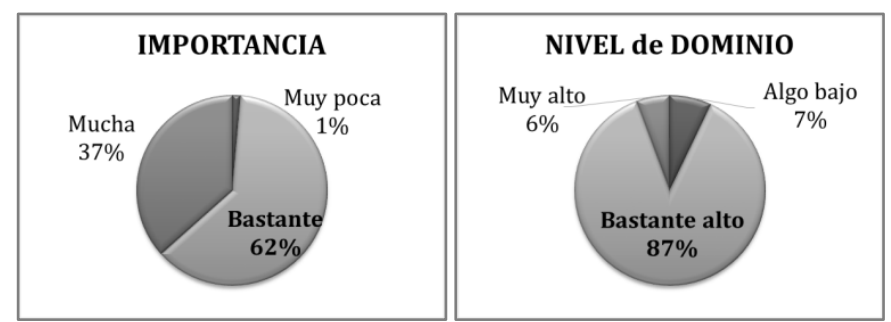

Figura 1. Importancia y nivel de dominio de la competencia de trabajo en equipo

En resumen, el perfil mayoritario de los participantes en este estudio es el de un hombre, matriculado por segunda vez en la asignatura, que ha realizado hasta doce trabajos durante sus estudios universitarios, que admite que la competencia de trabajo en equipo es bastante o muy importante, y que considera que domina las habilidades para trabajar en equipo en un nivel bastante alto.

En la siguiente sección se exponen los resultados del análisis de las respuestas a las preguntas relacionadas con los factores del trabajo en equipo considerados en este estudio.

\section{Resultados}

A continuación se exponen los resultados del cuestionario que contestaron los participantes, pregunta a pregunta. Además, se comparan algunos de esos resultados con los obtenidos en los trabajos de Florentín (2016), Lerís et al (2014) y Viles, Zárraga y Jaca (2013), también basados en el modelo IMO. Y se deja la discusión en relación a los dos 
problemas enunciados en la sección de contexto para las conclusiones.

\section{A. La evaluación de los trabajos en equipo}

Las distribuciones de las respuestas dadas a la pregunta 7 , en relación a cuatro aspectos de la evaluación del trabajo en equipo durante los estudios universitarios, se encuentran en la tabla 1; en ella se han destacado las valoraciones de máxima frecuencia.

Aunque, esas modas, de grado de acuerdo, coinciden en las dos primeras afirmaciones y también son iguales las de las otras dos, no son suficientemente representativas de la opinión de los participantes. El panorama se puede visualizar con más nitidez en la figura 2, en la que están representados los odds o cocientes entre la suma de frecuencias de las dos valoraciones superiores (bastante y totalmente de acuerdo) y la suma de frecuencias de las dos valoraciones inferiores (poco y algo de acuerdo). Se observan tres ideas:

- $\quad$ Es prácticamente igual de frecuente que un participante opine que el trabajo en equipo está valorado adecuadamente como lo contrario (odd de E1 es 1.09).

- Por cada diez estudiantes que opinan que la evaluación individual del trabajo en equipo aparece en pocas ocasiones, ocho opinan lo contrario (odds de E2 y de E4 es 0.78 y 0.82 , respectivamente).

- En cambio, es la mitad de frecuente que un participante esté bastante o totalmente de acuerdo en que se utiliza la autoevaluación (odd de E3 es 0.54) en la calificación del trabajo en equipo.

Tabla 1

Distribución del grado de acuerdo con las afirmaciones sobre la evaluación del trabajo en equipo

\begin{tabular}{llll}
\multicolumn{4}{c}{ Grado de acuerdo } \\
\hline Nada & Algo & Bastante & Totalmente
\end{tabular}

E1. El profesorado le asigna al trabajo de grupo un peso adecuado en la calificación final de la asignatura.

$$
9 \text { (12.7\%) } 25 \text { (35.2\%) } 30 \text { (42.3\%) } 7 \text { (9.9\%) }
$$

E2. El profesorado distingue en la evaluación los diferentes niveles de participación de cada uno de los miembros del equipo

$$
14(19.7 \%) \quad 26(36.6 \%) \quad 27 \text { (38\%) } \quad 4 \text { (5.6\%) }
$$

E3. El profesorado utiliza la autoevaluación de cada alumno/a en la calificación del trabajo.

$$
15(21.1 \%) \quad 31 \text { (43.7\%) } 18 \text { (25.4\%) } 7 \text { (9.9\%) }
$$

E4. El profesorado tiene en cuenta, para la calificación final del trabajo, las evaluaciones realizadas por los miembros del grupo entre sí.

$$
10(14.1 \%) \quad 29(\mathbf{4 0 . 8 \% )} \quad 27(38 \%) \quad 5(7 \%)
$$

En relación a la afirmación E2 sobre la individualización en la evaluación del trabajo en equipo, cabe señalar la mejora que supone que el $43.6 \%$ de los participantes en este estudio digan que están bastante o totalmente de acuerdo con esa afirmación, frente a tan sólo el 27\% del estudio de Lerís et al (2014), realizado con jóvenes recién incorporados a grados de Ingeniería.

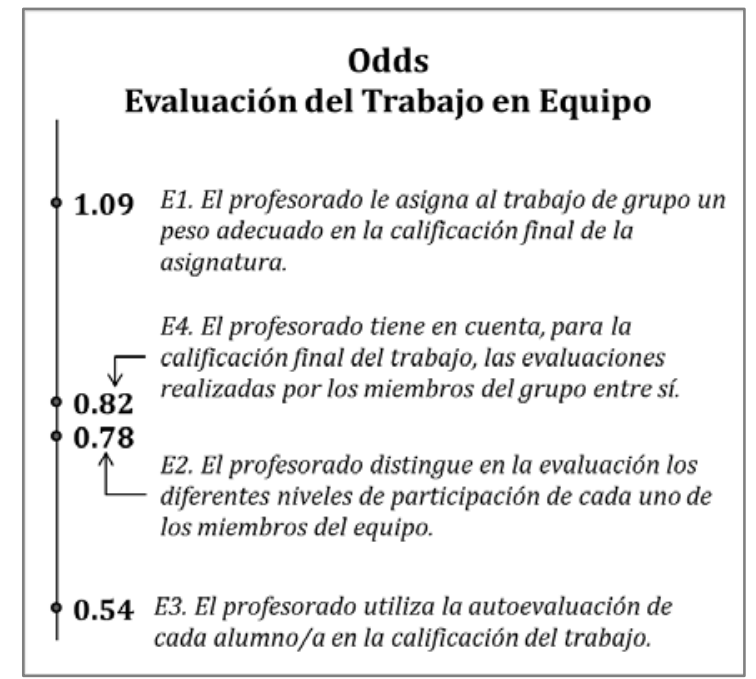

Figura 2: Odd de cada una de las afirmaciones sobre la evaluación del trabajo en equipo

\section{B. La composición del equipo.}

Sobre este asunto, el estudio del trabajo en equipo en los grados de maestro en la Universidad de Zaragoza, realizado por Florentín (2016), muestra la indecisión, tanto del profesorado como del alumnado, por quienes deberían ser los responsables de construir los equipos de estudiantes y con qué criterios.

En nuestro estudio, los participantes contestaron en la pregunta 8 cuál sería, a su juicio, el método más adecuado para formar equipos durante sus estudios universitarios. Las frecuencias de respuesta a las opciones ofrecidas se muestran en la tabla 2.

Destaca que lo menos aceptado por los participantes es la utilización de criterios académicos para formar equipos, independientemente de si es realizada por el alumnado o el profesorado (opciones de respuesta 2 y 3). Como era de esperar el criterio preferido es el de amistad (opción 1). Si bien, nos ha resultado sorprendente que esté seguido muy de cerca por la opción que propugna que el equipo tenga una composición diversa (opción 4), como, por ejemplo, el de la heterogeneidad de los estilos de aprendizaje (Lerís, Letosa, Usón, Allueva y Bueno, en prensa) o el de señalar previamente un líder (Viles et al., 2013).

Tabla 2

Método para la formación de equipos

Selecciona el método que, a tu juicio, se debería seguir para la formación de equipos durante los estudios universitarios

Opciones de respuesta

Frecuencia Porcentaje

\begin{tabular}{lcc}
\hline 1. Debería realizarla el alumnado & $\mathbf{3 0}$ & $\mathbf{4 2 . 2 5 \%}$ \\
aplicando criterios de amistad & & \\
2. Debería realizarla el alumnado & 12 & $16.90 \%$ \\
aplicando criterios académicos & & \\
3. Debería realizarla el profesorado & 5 & $7.04 \%$ \\
aplicando criterios académicos. & & \\
$\begin{array}{l}\text { 4. Debería tener una composición } \\
\text { diversa de los miembros del grupo } \\
\text { (edad, sexo, formación, } \\
\text { experiencias...) }\end{array}$ & $\mathbf{2 4}$ & $\mathbf{3 3 . 8 0 \%}$ \\
\end{tabular}




\section{La normativa del equipo}

Los participantes fueron preguntados por su opinión respecto a la existencia de normas de funcionamiento de un equipo durante los estudios universitarios, y también quienes deberían ser los responsables de generarlas (pregunta 9). Las frecuencias de cada una de las cuatro opciones ofrecidas son las siguientes:

- $\quad$ No debe existir ninguna norma: 5 (7\%).

- Deben existir normas, pero establecidas por el alumnado: $20(28 \%)$.

- Deben existir normas, pero establecidas por el profesorado: 7 (10\%).

- Deben ser negociadas entre el profesorado y el alumnado: 39 (55\%).

Merece ser destacado que los participantes consideran, de forma casi unánime (93\%), que sí deberían existir normas que regulen el funcionamiento de los equipos, lo cual está en completa sintonía con lo observado por Florentín (2016). La diferencia surge cuando se pregunta quién debería ser el responsable de establecer esas normas. En efecto, por cada participante que lo dejaría en manos del profesorado, casi tres opinan que lo adecuado sería que las estableciera el alumnado y más de cinco participantes preferirían que se negociaran entre los dos agentes, el alumnado y el profesorado.

\section{El funcionamiento del equipo}

En la pregunta 10 se requiere a los participantes que indiquen su grado de acuerdo con cuatro afirmaciones sobre el funcionamiento del equipo, la primera de ellas sobre un aspecto de los procesos de transición (F1) y las otras tres sobre elementos del desarrollo de la tarea (F2, F3 y F4).

En la tabla 3 se muestran las frecuencias (absolutas y porcentuales) de los grados de acuerdo con cada una de las frases incluidas en la pregunta; de nuevo, están resaltadas en negrita las opciones con mayor frecuencia de respuesta. Los datos de esa tabla 3 ya permiten apreciar diferencias importantes en la distribución de las frecuencias de las dos primeras afirmaciones respecto de las dos segundas, pues, por ejemplo, los valores de las modas son diferentes.

\section{Tabla 3}

Frecuencia de respuesta a la pregunta 10

Según tu experiencia previa en los trabajos en equipo durante los estudios universitarios, indica cuál es tu grado de acuerdo con las siguientes afirmaciones sobre el funcionamiento del equipo

\section{Grado de acuerdo}

\begin{tabular}{|c|c|c|c|}
\hline Nada & Algo & Bastante & Totalmente \\
\hline \multicolumn{4}{|c|}{$\begin{array}{l}\text { F1. El equipo suele establecer las consecuencias que tendría para los } \\
\text { participantes no cumplir los compromisos asumidos. }\end{array}$} \\
\hline $10(14.1 \%)$ & $24(33.8 \%)$ & $30(42.3 \%)$ & \\
\hline \multicolumn{4}{|c|}{$\begin{array}{l}\text { F2. El papel de coordinador o portavoz es positivo en la organización } \\
\text { del equipo. }\end{array}$} \\
\hline $3(4.2 \%)$ & $30(42.3 \%)$ & $21(29.6 \%)$ & 17 (23.9\%) \\
\hline \multicolumn{4}{|c|}{$\begin{array}{l}\text { F3. La carga de trabajo se reparte equitativamente entre las personas } \\
\text { del equipo, es decir, todos trabajamos por igual. }\end{array}$} \\
\hline $6(8.5 \%)$ & 15 (21.1\%) & 24 (33.8\%) & $26(36.6 \%)$ \\
\hline \multicolumn{4}{|c|}{$\begin{array}{l}\text { F4. Tomamos decisiones, de forma consensuada, para garantizar la } \\
\text { coherencia global del trabajo de equipo. }\end{array}$} \\
\hline 0 & $11(15.5 \%)$ & $35(49.3 \%)$ & 25 (35.2\%) \\
\hline
\end{tabular}

Si bien, quedan más claras las diferencias en la figura 3, en la que están representadas las odds de los dos niveles superiores de acuerdo respecto a los dos inferiores y, además, salen a la luz otras peculiaridades.

En efecto, podemos afirmar que, por un lado, la gestión de las personas jetas y pasotas (F1) y la coordinación del equipo (F2) son los que han sido puntuados más bajo por los participantes. De hecho, en ambos casos, es casi tan frecuente que señalen los dos niveles superiores de acuerdo como los dos inferiores, pues las correspondientes odds toman los valores 1.09 y 1.15, respectivamente, muy cercanos a 1 .

Por otra parte, el reparto de tareas (F3) es más frecuente que se realice de forma equitativa. Y, quizás lo más revelador es la práctica muy frecuente de tomar decisiones consensuadas (F4).

\section{Odds - Funcionamiento de los Equipos}

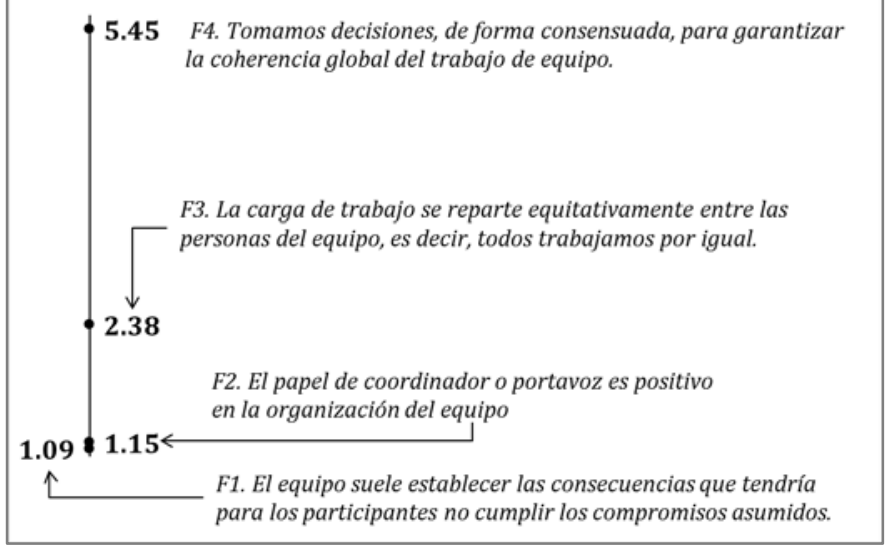

Figura 3; Odds de algunos aspectos de funcionamiento de los equipos.

Si dirigimos la atención a la afirmación F2 respecto al rol de coordinador, Lerís et al. (2014) analizaron si: “alguna persona del grupo solía realizar un seguimiento del desarrollo del trabajo para identificar incidencias y hacer cumplir la planificación” (p. 16) y observaron que se producen diferencias importantes entre los resultados del pretest y del postest si, en el ínterin, los participantes son formados y requeridos para realizar ese tipo de tareas.

Por otro lado, Viles et al. (2013) encontraron que los participantes tras su experiencia de trabajo en equipo, basada en el modelo IMO, optaban por una descripción de un líder interno entre estos dos niveles:

3. El líder se asegura de que cada miembro del equipo conoce y asume sus tareas y responsabilidades, y hace un seguimiento de los resultados que van obteniendo.

4. Además muestra su compromiso personal y entusiasmo, provoca intelectualmente a su gente, escucha, delega y da luego feedback constructivo a los miembros del equipo. (p. 304)

Por tanto, si bien el seguimiento del progreso de las tareas del equipo no es una función que sea asumida de forma espontánea por algún miembro del equipo, ambos estudios (Lerís et al., 2014, Viles et al., 2013) indican que puede modificarse ese comportamiento cuando el profesorado da formación y soporte para la realización de esa función. 


\section{E. El seguimiento externo de las tareas.}

Con la pregunta 11: indica cuál es tu opción preferida sobre la planificación del trabajo en equipo por parte del profesorado, se pretende averiguar la percepción de los estudiantes sobre su capacidad para planificar y controlar la ejecución de las tareas del equipo a través de un sencillo indicador: su necesidad de que el profesorado intervenga y revise su desempeño. En la tabla 4 aparecen las frecuencias, absolutas y porcentuales, de cada una de las tres opciones de respuesta ofrecidas a la pregunta. Los resultados muestran que propugnan la realización de revisiones/tutorías intermedias; si bien, prefieren, dos a uno, que sean los propios equipos los que planifiquen el momento de esas revisiones con el profesorado.

\section{Tabla 4}

Seguimiento de las tareas grupales por el profesorado

Indica cuál es tu opción preferida sobre la planificación del trabajo en equipo por parte del profesorado.

\section{Opciones de respuesta}

1. Establecer revisiones parciales del trabajo obligatorias para todos los equipos.

2. Realizar revisiones parciales del trabajo, no obligatorias, a petición de cada uno de los equipos.

3. Sin realizar revisiones parciales del trabajo, primando el trabajo autónomo de cada equipo.
Tabla 5

Frecuencia de respuesta a la pregunta 12

Indica tu grado de acuerdo con las siguientes afirmaciones sobre tu aprendizaje con los trabajos en equipo que has realizado durante tus estudios universitarios.

\begin{tabular}{cccc}
\multicolumn{3}{c}{ Grado de acuerdo } \\
\cline { 2 - 3 } & Algo & Bastante & Totalmente \\
\hline
\end{tabular}

A1. Mi interés por la materia estudiada aumenta al realizar trabajos en equipo. (Motivación)

7 (9.9\%) $\quad 30$ (42.3\%) 28 (39.4\%) 6 (8.5\%)

A2. He aprendido y comprendido los contenidos tratados en esos trabajos. (Contenidos)

$$
0 \quad 14(19.7 \%) \quad \mathbf{4 8}(\mathbf{6 7 . 6 \% )} \quad 9(12.7 \%)
$$

A3. He comprendido cómo ha de trabajar un equipo eficaz. (Habilidades)

$$
2(2.8 \%) \quad 18(25.4 \%) \quad 38 \text { (53.5\%) } \quad 13(18.3 \%)
$$

A4. Me ha ayudado a sentirme parte activa de mi propio proceso de aprendizaje. (Actitudes)

$$
1(1.4 \%) \quad 21(29.6 \%) \quad 32(\mathbf{4 5 . 1 \% )} \quad 17(23.9 \%)
$$

Con el fin de resaltar las diferencias entre las tres últimas variables de aprendizaje, en la figura 4 están representadas gráficamente las odds de los dos niveles de respuesta superiores respecto a los dos inferiores. De este modo es posible observar la predominancia de la odd de la afirmación sobre el aprendizaje de contenidos (A2), frente a la de habilidades para trabajar en equipo (A3) o a la de mejora de actitudes (A4).

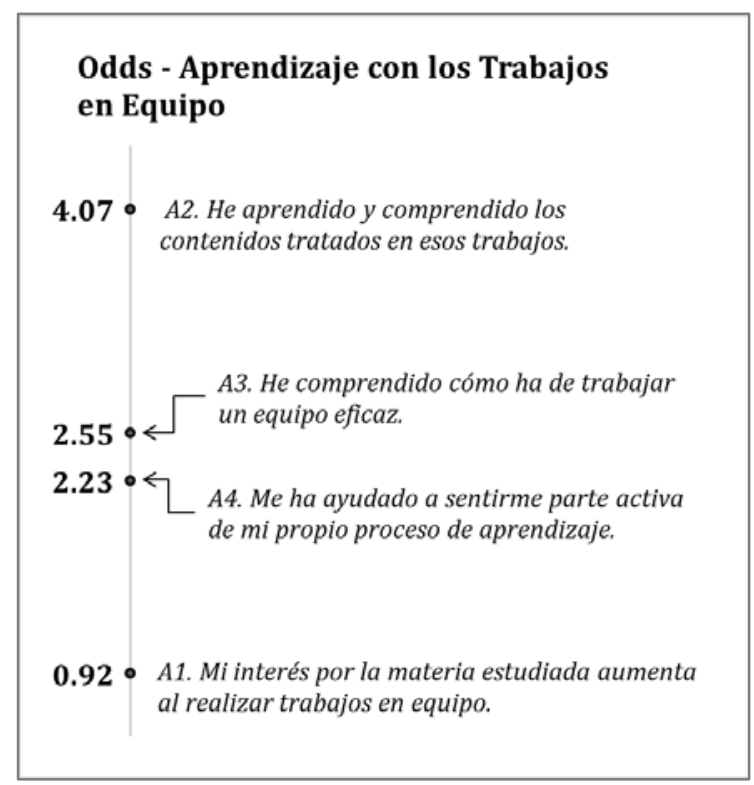

Figura 4: Odds de los cuatro tipos de resultados de aprendizaje

\section{CONCLUSIONES}

En el estudio realizado, hemos puesto el foco de atención en dos problemas del trabajo de un equipo, que, a nuestro juicio, es básico afrontar desde los inicios de la formación universitaria, independientemente del grado y de la asignatura. Son la responsabilidad individual y la planificación de tareas del equipo.

En la búsqueda de soluciones, se ha utilizado un marco teórico de la competencia de trabajo en equipo, que pone el 
énfasis en los comportamientos que hacen eficaz a un equipo (modelo IMO). Se han seleccionado los factores y actuaciones que abordan los mencionados problemas y se han sometido a la opinión de los estudiantes. Los resultados obtenidos conducen a una serie de sugerencias que exponemos a continuación.

En cuanto a la mejora de la responsabilidad individual, se han considerado dos factores de entrada, la evaluación y la cohesión grupal, y un proceso de transición del equipo, la normativa de funcionamiento. La percepción del alumnado nos lleva a recomendar al profesorado estos tres aspectos:

- Diferenciar la evaluación individual y la grupal, siendo necesario informar y formar a los estudiantes en las estrategias de coevaluación y autoevaluación.

- Dejar al alumnado que forme los equipos, aplicando criterios de amistad, al menos al inicio de los estudios de grado. No obstante, los participantes han señalado que les parece igualmente adecuado formar equipos basados en la diversidad de las características de sus miembros. Cabe esperar, por tanto, que crear grupos heterogéneos podría ser comprendido y aceptado por el alumnado.

- Establecer normas de funcionamiento para los equipos, que sean consensuadas con el profesorado. Quizás esta preferencia del alumnado se deba a que el equipo necesita la complicidad con el profesorado para el cumplimiento de algunas de esas normas.

En síntesis, podemos decir que el diseño del trabajo en equipo en los primeros cursos de grado podría ayudar a mejorar la responsabilidad individual de sus miembros, si el profesorado aplica algún método de evaluación individualizada (siempre que los estudiantes sea instruidos previamente en ella), si permite que los estudiantes formen sus propios equipos, o se aplican criterios de diversidad, y si les ayuda a establecer unas normas de equipo.

Por otro lado, a nuestro juicio, el problema de la ejecución de un plan de trabajo se debería abordar desde dos frentes: el interno al equipo y el externo. El estudio realizado muestra que, en los primeros cursos del grado, los estudiantes han de ser formados para aprender a ejercer de coordinadores, al menos, en lo que se refiere a realizar el seguimiento de un sencillo plan de trabajo. La unanimidad de los participantes en apoyar las revisiones intermedias del trabajo por el profesorado revela que necesitan de ese liderazgo externo, que podría ser complementado por el liderazgo interno para el que se estaría formando a los coordinadores.

En definitiva, sugerimos que el profesorado establezca uno o varios periodos en los que se revisan conjuntamente los logros del equipo. Quizás podrían añadirse tareas a cargo del coordinador, como registrar la actividad del equipo o rendir cuentas del cumplimiento del plan de trabajo.

Finalmente, queremos añadir que formar a nuestros jóvenes en la competencia de trabajo en equipo es una labor compleja, en gran medida debido a la multidimensionalidad del concepto. Es, por ello, que es necesario seguir indagando sobre programas y modelos formativos sencillos, aplicables y eficaces en el contexto universitario español, sin olvidar la necesidad de que esa formación sea progresiva y sostenida.

\section{AGRADECIMIENTOS}

Agradecemos al Gobierno de Aragón y al Fondo Social Europeo el soporte para la realización de este trabajo. También queremos expresar nuestra gratitud al grupo de investigación GIDTIC (http://gidtic.com) del que formamos parte.

\section{REFERENCIAS}

Florentín, P. (2016). La competencia de trabajo en equipo en la Facultad de Educación de la Universidad de Zaragoza: análisis y estrategias para su mejora (Tesis doctoral). Universidad de Zaragoza, Zaragoza, España.

García, M.M., González, I y Mérida, M. (2012). Validación del Cuestionario de Evaluación ACOES. Análisis del trabajo cooperativo en educación superior. Revista de Investigación Educativa, 30(1), 87-109.

Lerís, D., Fidalgo, A. y Sein-Echaluce, M. L. (2014). A comprehensive training model of the teamwork competence. International Journal of Learning and Intellectual Capital, 11 (1).

Lerís, D., Letosa, J., Usón, A., Allueva, P. y Bueno, C. (En prensa). Trabajo en equipo y estilos de aprendizaje en la educación superior. Revista Complutense de Educación.

Mathieu, J., Maynard, M. T., Rapp, T. \& Gilson, L. (2008). Team Effectiveness 1997-2007: A Review of Recent Advancements and a Glimpse Into the Future. Journal of Management, 34(3), 410-476.

doi: http://doi.org/10.1177/0149206308316061

Rousseau, V., Aubé, C. \& Savoie, A. (2006). Teamwork Behaviors: A Review and an Integration of Frameworks. Small Group Research, 37(5), 540-570. doi: http://doi.org/10.1177/1046496406293125

Torrelles, C., Coiduras, J., Isus, S., Carrera, F. X., París, G. y Cela, J. M. (2011). Competencia de trabajo en equipo: Definición y Categorización. Profesorado: Revista de currículum y formación del profesorado, 15(3), 329-344.

Viles, E., Zárraga, M. y Jaca, C. (2013). Herramienta para evaluar el funcionamiento de los equipos de trabajo en entornos docentes. Intangible Capital, 9(1), 281-304. 